\title{
EVALUATION OF A RAPID AND EFFICIENT METHOD FOR PREPARATION OF SKELETONS OF RABBIT AND GOOSE
}

\author{
M. T. Mussa ${ }^{1 *}$, M. M. Kamal ${ }^{2}$, M. A. A. Mahmud ${ }^{3}$, B. K. Sarker ${ }^{4}$, M. A. Jalil ${ }^{5}$ and S. K. Das ${ }^{2}$ \\ ${ }^{1}$ Department of Anatomy and Histology, Jhenaidah Government Veterinary College, Jhenaidah-7300, Bangladesh; \\ ${ }^{2}$ Department of Anatomy and Histology, Bangladesh Agricultural University, Mymensingh-2202, Bangladesh; \\ ${ }^{3}$ Department of Anatomy and Histology, Bangabandhu Sheikh Mujibur Rahman Agricultural University, Gazipur- \\ 1706, Bangladesh; ${ }^{4}$ Department of Pathology and Parasitology, ${ }^{5}$ Department of Medicine and Surgery, Jhenaidah \\ Government Veterinary College, Jhenaidah-7300, Bangladesh
}

\begin{abstract}
The primary objective of this study was to prepare the skeletons of rabbit and goose by rapid, economic and efficient method. Total 10 (5 rabbits and 5pink-footed goose) matured rabbits (Oryctolagus cuniculus) and pink-footed geese (Anser brachyrhynchus) were used. At first, the killed animals were skinned and defleshed with the help of scalpel and boiled in $3 \%$ solution of soda water for 1 hour. After cooling, the bony samples were cleaned and dipped into 10\% solution of bleaching water for 2 hours and then were washed in tap water. Finally, the bony segments were articulated for rising into skeleton after complete sundry. The whole processes were taken a day (15-20 hours). This method is very effective because there is no chance of losing small bones, requires less expenditure, time and labour. It is also mentionable that the original color of the bone can be maintained and the visceral organs can be used for other studies.
\end{abstract}

Keywords: Skeleton, rapid, efficient, method, preparation, rabbit, goose

\section{INTRODUCTION}

Skeleton is called the mirror of the body that supports, protects and forecasts the body structure of an organism. It can be defined as a hard framework of the body which supports soft structures. This is composed of bones, cartilages and ligaments. The skeleton is responsible for the maintenance of the shape flexibility as well as locomotion of the body. For the purpose of description, it is divided into two parts, Axial and Appendicular skeletons. The axial skeleton comprises of the bones of skull, vertebral column, ribs and sternum. The appendicular skeleton is made up of the bones of the limbs (Ghosh, 2006). Collections of animals and birds include a variety of preparation types emphasizing preservation of feathers, skeletons, soft tissues, or some combination thereof (Wikipedia, 2010; Winker, 2000).

Skeletons of animals and birds are the basic need of the veterinary study, especially for the veterinary gross anatomy. These skeletons are necessary for research ranging from phylogenic investigations to age and growth analyses to functional morphology (Bemis et al., 2004; Burke and Feduccia, 1997; Olson, 1973) and essential tools for the study of systematic, biomechanics, evolutionary morphology \& adaptation, paleontology and identification of animal remains from archeological sites (http://research.calacademy.org/om/collections/prep skeletal). Vertebrate skeletons or parts of skeletons are preserved in museums, research institutes, organizations for the protection of nature and in schools for research and teaching (Bemis et al., 2004). Skeleton collections are likewise essential for studies of the food remains of predatory birds and mammals; these have been used as indices of body size and in physiological or histological studies (Gofur and Khan, 2010). These are also used to identify animals and birds as well as in various forensic applications, such as the identification of carcasses of illegally taken animals and birds or, in rare cases, bones that have been taken as evidence in other crimes (Olson, 2003). Large series of skeletons provide a sound basis for assessing geographic size variation within a species and it is also essential for the identification of fossils, bones from archaeological sites and food items taken by predatory animals, although world skeletal inventories have greatly aided researchers, they also reveal serious deficiencies in museum holdings (Olson, 2003). So, necessity of preparing skeleton of animals and birds is increasing rapidly.

*Corresponding e-mail address: t.mussadvm@gmail.com 


\section{T. Mussa and others}

Most of the anatomists follow the traditional methods of preparation of the skeleton. It usually takes 1 to 2 months depending upon the size of the animals (Gofur and Khan, 2010). Raghavan (1964) describes the traditional method of preparation of skeleton with maceration which may be required about 1 to 2 months. Selby (1987) describes a simple three-day technique for preparing skeleton of adult mice. A few anatomists have tried to develop a short and easy technique, yet 3 days to 2 weeks time is required for raising a complete cleared skeleton. In this experiment, the aim was to develop a quick, economic and efficient method for preparation of skeleton of small animals and birds (Gofur and Khan, 2010). The present study was undertaken to investigate the rapid, economic and effective method of preparation of skeletons of rabbits and birds.

\section{MATERIALS AND METHODS}

A total of 10 matured rabbits (Oryctolagus cuniculus) and geese (Anser brachyrhynchus) were used in this experiment. Five rabbits and 5 geese were collected from local market in Jhenaidah sadar. Care should be required during preparing animal skeletons where health and safety are concerned. So rubber gloves during preparing a carcass and care was taken to avoid stick injuries following the concept of Baker et al. (2003).

\section{Animals and birds preparation}

The rabbits and goose were kept in fasting condition for 24 hours. The rabbits were killed by intravenous injection of saturated magnesium sulphate $\left(\mathrm{MgSO}_{4}\right)$ solution @ $2 \mathrm{ml} / \mathrm{kg}$ body weight. In case of some goose, $\mathrm{MgSO}_{4}$ was injected directly into heart and some also were killed by cervical subluxation (disarticulation of atlanto-occipital joint \& puncture of occipital artery). These methods and procedures of killing rabbits and goose were approved by the IACUC for humane killing of animals and same as the recommendations of the American Veterinary Medical Association (AVMA) Panel on Euthanasia, 2000 and in accordance with humane euthanasia as defined by the Federal Animal Welfare Act (54 FR 36112-36163) (http://research.uiowa.edu/animal).

\section{Skinning and defleshing}

The skin and muscles, adipose tissue and all visceral organs were removed with the help of scalpel and blades, forceps, scissors, knife up to maximum extent as possible so that the bones were visible.

\section{Decomposing the carcass}

The bones of the rabbit and goose were boiled in $3 \%$ solution of soda water for 1 hour separately. Then it was kept for 2 hours for cooling and 3 hours for cleaning according to the concept of Baker et al. (2003) and Van Cleave (2010). Soda (Sodium carbonate, $\mathrm{Na}_{2} \mathrm{CO}_{3}$ ) water solution was used for proper and complete digestion of muscles (Gofur and Khan, 2010) and that will help to remove muscles and related structures easily.

\section{Sequential arrangement of bones}

After boiling in soda water these bony segments were taken out and again the left over flesh and tissue on the bones were scratched by BP handle and blade as maximum as possible. Then the ribs were gently pulled away the backbone. The vertebrae and ribs were placed in order on a sheet and secured with the help of fine stainless steel pins. It was vital subject to keep the bones in order and in exact position where the ribs attached along with the backbone and then the bones were washed and cleaned by normal water.

\section{Bleaching and drying}

The bones of goose and rabbits were taken to dip into $10 \%$ bleaching water solution for 2 hours to avoid further destruction by microorganisms and then the bony segments were allowed for complete sun drying for 10 hours.

\section{Arrangement of bones into skeleton}

In this time bones were articulated in proper position into the skeletons. The ribs of rabbits and goose were attached with vertebrae by small, thin and non-corrosive wires. A small hole was drilled by drill machine through the head of each rib and the corresponding attachment points on each vertebra. Each pair of ribs was bind with corresponding vertebra with the help of short wire through these holes. After attaching all the ribs to the 
backbone, each vertebra was threaded, in order, onto a strong wire that was slightly longer than the overall length of animals. A long (skull to coccygeal vertebral canal) strong rod was insert into the vertebral canals so that it can support the weight of the skeleton. The tail and body wires were then joined together, leaving enough wire protruding forward from the neck, to allow attachment of the skull later according to Gofur and Khan (2010). In case of birds, the bones were articulated with wire where possible; otherwise, the bones were articulated with glue following the concept of Van Cleave (2010).

\section{Positioning the skeleton}

A table was used for positioning the skeleton and the skeleton was draped over this in a life-like pose. Finally, the skeletons were housed in display cases to prevent dust from inside and outside of the building.

\section{RESULTS AND DISCUSSION}

Skeleton is composed of bones, cartilages and ligaments and is responsible for the maintenance of the shape flexibility as well as locomotion of the body (Ghosh, 2006). It also levers the soft structures of the body. According to Getty (1975), skeleton is the framework of hard structure which supports and protects the soft tissues of animals and birds.

Formulation of skeleton is very important for anatomical study. Preparation of animal skeletons, contrary to what many people imagine, is a technical issue and really not too smelly (Baker et al., 2003). Systematic studies in the various branches of vertebrate zoology differ fundamentally according to differences in traditional methods of specimen preparation (Olson, 2003). The time requirement varies according to the method adaptation for preparation of skeleton. Adult and mature rabbits and goose were used in this experiment, to avoid the risk of softening and shrinkage of bones because boiling can shrink bones (Van Cleave, 2010). The animals and birds were not fixed because fixation prolongs the time of treatment but does not improve the quality of skeleton preparation (Green, 1952). According to the objective of the study was to prepare skeleton within a very short time and efficiently, after skinning as much flesh as possible were removed without risking damage to delicate parts of skeleton. Once the crudely defleshed carcass was obtained, the next step was decomposing carcass (Gofur and Khan, 2010). It is usually done by remaining the carcass in a pit for 1-2 months according to the size of the animals and birds (Raghavan, 1964). This process of skeleton preparation is time consuming, laborious and expensive also. As this process can easily be done even at home without full laboratory facilities by using proteolytic enzymes like neutrase and also by using biological washing powders or meat tenderizers (Baker et al., 2003), soda water was used to decompose carcass. Although Baker et al. (2003) does not suggest to use of biological washing powder to decompose carcass as it is corrosive and can damage bone, so in this study we used soda water as our aim was to prepare the skeleton quickly as sodium hydroxide is frequently used in the process of decomposing road kill dumped in landfills by animal disposal contractors (Leon and Justin, 2004); on the other hand use of proteolytic enzyme like neutrase is expensive (Baker et al., 2003). The bones of the rabbit and goose were separately boiled in $3 \%$ solution of soda water for 1 hour (Table 1) using normal heater instead of incubator. Decomposing and cleaning the flesh from bones by boiling is not preferred if quality specimens are needed because boiling can shrink bones but the negative effects of boiling are negligible compared to how easy and inexpensive this bone cleaning process (Van Cleave, 2010). After boiling as well as decomposing the carcass, 2 hours time was required for clearing the carcass (removing the tenderized meat over the bones) and then bleaching to avoid further decomposition by microorganisms because bones generally contain a lot of grease. The grease can cause problem such as over the years, grease can breaks down as a result of bacterial decay and the resulting acids attack the bones and bones become unpleasant and smelly (Baker et al., 2003). For this, the bones were dipped in 10\% bleaching water solution for 3 hours for geese and rabbit (Table 1). After bleaching then the bones were dried absolutely (Table 1) by sun rays. Time saving and economical skeleton preparation is the main objective, so in this process every steps requires less time, labour inexpensive and kept the bones less time in bleaching water solution. If there may remains intractable bits of gristle attached or a few materials remain inside some cavities, and then may raise a question of bacterial activity (Gofur and Khan, 2010). But absolutely dry the bones, it might mummify and bacterial activity will cease (http://www.skullsite.co.uk/preparation.htm). Then the bones were articulated to rise into skeletons and each skeleton was positioned on a suitable base with the help of two rods and thick wire (Figs. 1 and 2). Finally all skeletons were housed in a display case. The total time requirement for the preparation of skeleton in this process 


\section{T. Mussa and others}

is 1day (15-20 hrs) for geese and rabbit, the time variation was occurred according to the size of animals and birds.

Table 1. Steps involving in preparing skeleton of small animals and birds

\begin{tabular}{|c|c|c|c|}
\hline \multicolumn{2}{|c|}{ Steps involving for skeleton preparation } & \multicolumn{2}{|l|}{ Time requirements } \\
\hline \multicolumn{2}{|c|}{ Skinning and removing flesh and viscera } & As quick as possible & As quick as possible \\
\hline \multirow[t]{2}{*}{ Decomposing carcass } & Boiling & 1 hour & 1 hour \\
\hline & Cooling & 2 hours & 2 hours \\
\hline Clearing & & 3 hours & 3 hours \\
\hline Bleaching & & 2 hours & 2 hours \\
\hline Drying & & 10 hours & 10 hours \\
\hline Assembling the bones & & As time required & As time required \\
\hline
\end{tabular}

From the above table we may say this method of preparation of skeletons of rabbit (Fig. 1) and geese (Fig. 2), is very easy and rapid (Table 1) for completely cleaned and natural white shiny skeleton.

Most of the anatomists made skeleton by following the traditional method such as digging the grave and putting the animals into the pit and removing the bones out of the pit after 1 to 2 months depending upon the size of the animals, cleaning and preparation of the skeleton (Raghavan, 1964). The traditional method had some disadvantages as it is time consuming, chance of missing of bones especially small bones, bones may lose the natural color, fear of digging the grave by carnivores and the viscera cannot use for further study. On the other hand this method of preparation of skeleton has some advantages such as it is time saving (Table 1), all bones obtained easily, the visceral organs can be utilized for other studies if these are properly cleaned, washed and preserved in $10 \%$ buffered formalin, the expenditure, time and labor of digging the grave and again removing the bones from the grave can be saved and no chance for digging the grave by carnivores that advantages was also described by Gadre (2007). Another technique that is slightly better opportunity for preparation of skeleton, but requiring 1-2 weeks (Selby, 1987). So the economic, efficient and simple procedure (Table 1), with minor modifications, can be used to prepare the most skeletons of small animals and birds.

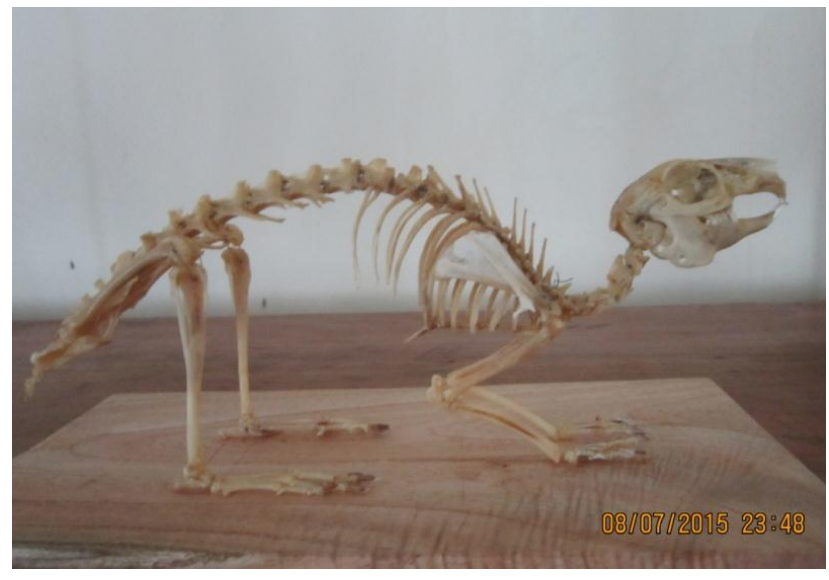

Fig. 1.Skeleton of rabbit

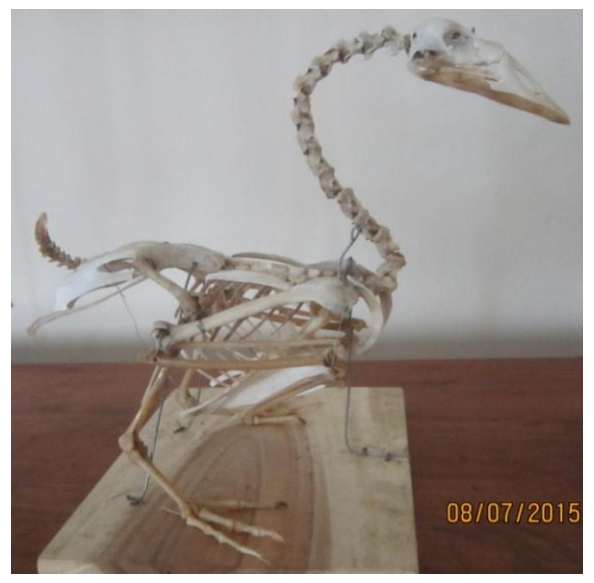

Fig. 2. Skeleton of Goose

Preparation of skeletons of rabbit and goose by rapid, economic and efficient method has proved very useful for the study of bones of small animals and birds. Advantages of this method are there is no chance of losing small bones, expenditure, time and labour can be saved, original color of the bone can be maintained and 
visceral organs can be used for further studies. Further study is required to improve this method for preparation of the skeleton of small animals and birds.

\section{ACKNOWLEDGEMENTS}

The authors are acknowledging to the Department of Anatomy and Histology, Jhenaidah Government Veterinary College for technical and financial supports.

\section{REFERENCES}

1. Baker P, Davis S, Payne S and Revill M (2003). On preparing animal skeletons - a simple and effective method. International Council for Archaeozoology Newsletter 4: 4 -15.

2. Bemis WE, Hilton EJ, Brown B, Arrindell R, Richmond AM, Little CD, Grande L, Forey PL and Nelson GL (2004). Methods for preparing dry, partially articulated skeletons of osteichthyans, with notes on making ride wood dissections of the cranial skeleton. Copeia 3: 603-609.

3. Burke AC and Feduccia A (1997). Developmental patterns and the identification of homologies in the avian hand. Science 278: 666-668.

4. Gadre KM (2007). Quick method of preparation for animal and bird skeleton. Indian Journal of Animal Sciences 77: 245-246.

5. Getty R (1975). General osteology. The Anatomy of the Domestic Animals. Vol-1, $5^{\text {th }}$ edn., W. B. Saunders Co., Philadelphia.

6. Ghosh RK (2006). Primary Veterinary Anatomy, $4^{\text {th }}$ edn., Current Book International, Kolkata.

7. Gofur MR and Khan MSI (2010). Development of a quick, economic and efficient method for preparation of skeleton of small animals and birds. International Journal of BioResearch 2: 13-17.

8. Green MC (1952). A rapid method for clearing and staining specimens for the demonstration of bone. The Ohio Journal of Science 52: 31-33.

9. http://research.calacademy.org/om/collections/prep skeletal (Access date: Feb, 2010).

10. http://research.uiowa.edu/animal (Animal Research, Institutional Animal Care \& Use Committee) (Access date: Feb, 2010).

11. http://www.skullsite.co.uk/preparation.htm; the site developed by the experimental zoology group of Wageningen University, Netherlands (Access date: Feb, 2010)

12. Leon TH and Justin K (2004). Carcass Disposal: A Comprehensive Review. Chapter 6. National Agricultural Biosecurity Center, Kansas State University, USA.

13. Olson SL (1973). Evolution of the rails of the South Atlantic Islands. Smithsonian Contributions to Zoology 152.

14. Olson SL (2003). Development and uses of avian skeleton collections. Bulletin BOC 123A: 26-34.

15. Raghavan D (1964). Anatomy of Ox. Indian Council of Agriculture Research, New Delhi.

16. Selby PB (1987). A rapid method for preparing high quality alizarin stained skeletons of adult mice. Stain Technology 62: 143-6.

17. Van Cleave J (2010). http://scienceprojectideasforkids.com/2010/how-to-prepare-a-chicken-skeleton (Access date: Feb, 2010).

18. Wikipedia, the free encyclopedia, http://en.wikipedia.org/wiki/Bird_collections (Access date: Feb, 2010).

19. Winker K (2000). Obtaining, preserving and preparing bird specimens. Journal of Field Ornithology 71: 250-297. 